\title{
The Cure Rate Modeling: An Application with Bayesian approach in Liver Cirrhosis Patients
}

\author{
Dilip C Nath ${ }^{1}$, Atanu Bhattacharjee ${ }^{2}$ and Ramesh K Vishwakarma ${ }^{3}$ \\ 1,3 Department of Statistics, Gauhati University, Guwahati-781014,India \\ 2 Division of Clinical Research and Biostatistics, Malabar Cancer Centre, Thalassery- \\ 670103, India.
}

Abstract: Survival analysis is the widely used statistical tool for new intervention com $\neg$ parison in presence of hazards of follow up studies. However, it is difficult to obtain suitable survival rate in presence of high level of hazard within few days of surgery. The group of patients can be directly stratified into cured and non-cured strata. The mixture models are natural choice for estimation of cure and non-cure rate estimation. The estimation of cure rate is an important parameter of success of any new intervention. The cure rate model is illustrated to compare the surgery of liver cirrhosis patients with consenting for participation HFLPC (Human Fatal Liver Progenitor Cells) Infusion vs. consenting for participation alone group in South Indian popula-tion. The surgery is best available technique for liver cirrhosis treatment .The success of the surgery is observed through follow up study. In this study, MELD (Model for End-Stage Liver Disease) score is considered as response of interest for cured and non-cured group. The primary efficacy of surgery is considered as covariates of interest. Distributional as $\neg$ sumptions of the cure rate are solved with Markov Chain Monte Carlo (MCMC) techniques. It is found that cured model with parametric approach allows more consistent esti-mates in comparison to standard procedures. The risk of death due to liver transplantation in liver cirrhosis patients including time dependent effect terms has also been explored. The approach assists to model with different age and sex in both the treatment groups.

Key words: MCMC, MELD score, Survival analysis, Posterior estimation

\section{INTRODUCTION}

Cirrhosis is special state of liver to be get trapped within the sea of scar and struggle to regenerate. It influences the gradual shrinkage of the size of the liver. In liver cirrhosis problem, the duration between transplantation to recover is crucial period for patients. Patients are generally measured through follow up periods with liver functioning effects. The disease severity in patient with liver cirrhosis is measured through the model for end stage liver disease (MELD) [1]. It is continuous disease severities scale with highly predictive of the risk of dying from liver. The score is adopted by UNOS (the United Network for Organ Sharing) for use in allocating livers to patients on the liver transplantation waiting list. Set of respondent are divided into two parts as cured and non-cured. The main goal of this work is to look on the cure and non-cured rate among liver cirrhosis patients through MELD score. The patients alive at any point of times are defined as cured otherwise non-cured. The mixture distribution is useful to formulate the cure rate model. The deep consideration is important on patients' treatment effects through long term observations. The model is ultimate choice for treatment effect comparison. It is widely applied in the field of economic, reliability and criminology. Recently, Cure rate model in medical research is elaborated and extended [1-8]. It is applied for AIDs in HIV positive patients [9]. The survival experience is defined with cure rate model. It is also used in the recidivism time among the prisoners in Western Australia [10]. The product of log-normal survival function is appropriate for non-cured portion of patients. The simple log-normal distribution as a choice of mixture model is elaborated in application to the analysis of important exploration in Japan [4]. It provides stratified results in drug 
treatment effect of lymphoma patients [11]. The exponential distribution as a choice of mixture model is useful for non-cured patients [12]. The MELD score (UNOS Modification) is calculated as follows

$$
\begin{aligned}
\text { MELD Score }= & 9.57 \times \operatorname{loge}(\text { serum creatinine })+3.78 \times \log e(\text { bilirubin })+11.2 \\
& \times \log e(I N R)+6.43 .
\end{aligned}
$$

The MELD is useful tool to detect the liver status in patients. It is widely used method for organ allocation in liver transplantation [13-15]. The score function is formulated with consideration of liver and renal functions. Several studies have concluded that the liver transplanted patients having low MELD score can be influenced for death [16-19]. The different biochemical parameters viz., serum creatinine, bilirubin \& INR are used to calculate the MELD score. The details to calculate the MELD score can be cited with

http://www.unos.org/docs/MELD_PELD_Calculator_Documentation.pdf.

In this study, the cure rate model is applied through the changes of MELD score among the liver cirrhosis patient. The computation of treatment effect is performed through survival analysis. The MELD score is applied as primary endpoint to perform the analysis. The data are considered from the phase 3 clinical trials in which liver cirrhosis patients were undergone with HFLPC for 36months. The dataset includes 104 patients, of whom 62 are male and 42 female.

\section{DATA METHODOLOGY}

Patients must have a MELD score of 12 to 24, were considered as ineligible for a solid liver transplant because of documented co-morbidities and must have an estimated life expectancy of approximately 6 to 18 months. The data considered from the path http://www4.stat.ncsu.edu/ boos/var.select/pbc.html, as accessed on Jan 28, 2013. Assessments for initial determination of patient eligibility (14 to 10 days prior to the day of intended cell transplantation) were included a complete physical examination and medical history, grading of encephalopathy, clinical laboratory studies ( haematology, blood chemistry, urinalysis and coagulation tests), a serum pregnancy test (for females of child-bearing potential), ABO blood typing (if not known), human leukocyte antigen(HLA)Class I antigen typing (if not known) and a Panel Reactive Antibody test with identification of any preformed antibodies, serologic testing for Epstein-Barr virus (EBV) and cytomegalovirus (CMV) and calculation of the patient's MELD. Only cells from an ABO-compatible donor with no HLA Class I antigen to which the recipient has performed antibodies were selected for transplant. Furthermore, cells from an EBV-positive or CMV-positive donor were only administered to EBV-positive or CMV-positive recipients.

Intravenous administration of adequate hydration was begun at 2:00 AM and last until about one hour before discharge. Assessments and procedures that was preceded the catheterization procedure include clinical laboratory studies, assessment of donor-specific HLA Class I antigens (expected to be absent prior to cell transplantation), grading of encephalopathy, a brief physical examination, calculation of the patient's MELD and Child-Pugh Scores and assessment of trough plasma tacrolimus concentration. Prophylactic antibacterial and antifungal agents were administered before (both agents) and after (antibiotics only)the catheterization procedure. Oral Pepcid (famotidine) and a 30-minute infusion of $250 \mathrm{mg}$ of Solu-Medrol (methylprednisolone) were administered 2 hours before splenic artery catheterization.

The patients were taken to the radiology suite approximately 30 to 60 minutes before splenic artery catheterization. After induction of conscious sedation, a catheter was inserted into the femoral artery and, under fluoroscopic guidance, passed into the splenic artery. The final position of the catheter was confirmed with a small volume of contrast dye. Blood pressure, heart rate, respiratory rate and $\mathrm{O} 2$ saturation was monitored frequently during cell infusion and until the 
catheter is removed. Confirmation of splenic artery and splenic vein patency with contrast media was performed just before cell infusion and again just after cell infusion.

Discharge medications were included Prograf (dosage adjusted, if necessary, based on assessment of trough tacrolimus concentration assessed that morning), prednisone (20 mg P.O. once daily for two days; then $10 \mathrm{mg}$ P.O. once daily for the next five days; then $5 \mathrm{mg}$ P.O. once daily until the Week 24 Visit) and Diflucan (fluconazole), $100 \mathrm{mg}$ P.O. once daily for ten days. Patients were scheduled to attend follow-up visits twice in the first week after cell transplantation, then weekly until eight weeks after cell transplantation, then once every four weeks until 24 weeks after cell transplantation, then once every three months until two years after cell transplantation. Routine clinical laboratory testing (haematology, blood chemistry and urinalysis) and assessments of trough plasma tacrolimus levels were also performed once each month between visits (that were scheduled every three months) after the Week 24 Visit. Efficacy assessments after cell transplantation was included the MELD Scores. Results of testing for donor-specific soluble antigens and data pertaining to episodes of encephalopathy were also contributed to the interpretation of efficacy in this study.

\section{PARAMETRIC CURE RATE MODEL}

Parametric cure rate model through Bayesian and Frequency approach are initiated nearly two decades earlier [20-22]. It is built with assumption of two groups of patients i.e. (I) cured and (II) non-cured. However, the distributional assumptions of group of patients are sometimes become problematic. Non-cured part is applicable for those samples that are subject to the event of death and rest of them are as cured. Those patients are survived till the end of the study is defined as cured group. The finite mixture of Weibull distributions [23] and Generalized Gamma distribution [2] in survival function has been elaborated in melanoma clinical trial data and found suitable. However, the models are quite complicated to apply for analysis. The flexible parametric model [24] in survival analysis is discussed and extended with relative survival function [23, 25]. The objective of this study is to show the effect of HFLPC on the liver cirrhosis patients through cure rate modelling. Let the observed failure time is $\mathrm{T}$. If $\mathrm{T}=\infty$, then patients are considered to be cured with new surgical procedure. Now, it is considered that $\mathrm{N}$ is the total number of patients in the study. For any value of $N,\left\{Y_{i}\right\}_{i=1}^{N}$ is assumed to be distributed with survival function

$$
P(Y>t)=S(t)=1-F(t)
$$

where $Y_{\mathrm{i}}$ is the survival time of ith individual in the study. Further, individuals not-cured are ordered as $\mathrm{Y}_{(1)}<\mathrm{Y}_{(2)}<\ldots, . . .,<Y_{(\mathrm{r})}<\ldots, .,<\mathrm{Y}_{(\mathrm{N})}$ through their survival time. The term $\mathrm{P}(\mathrm{Y}>t)$ is free from $N$. Let $r \leq N$ and the number of persons not-cured is $r$. Then $T=Y_{r}$ for $1 \leq r \leq N$. The conditional distribution of $\mathrm{T}$, given $\mathrm{N}$ and $\mathrm{r}$ is

$$
P(T \geq t \mid N, r)=V(N=0)+W(S(t) ; N-r+1, r) V(N \geq r \geq 1)
$$

where the term $\mathrm{V}$ is the indicator function.

Now,

$$
\begin{aligned}
& W(S(t) ; N-r+1, r)=\sum_{j=0}^{r-1}\left(\begin{array}{c}
N \\
j
\end{array}\right)[F(t)]^{j}[S(t)]^{N-j} \\
& W(S(t) ; N-r+1, r)=N\left(\begin{array}{l}
N-1 \\
r-1
\end{array}\right) \int_{0}^{S(t)} \mu^{N-r}(1-\mu)^{r-1} d \mu
\end{aligned}
$$

$\mathrm{W}(\mathrm{S}(\mathrm{t}) ; \mathrm{N}-\mathrm{r}+1, \mathrm{r})$ is the beta distribution function. The survival function with unconditional distribution is further defined as

$$
S^{*}(t)=W^{*}[P(T \geq t \mid N, r)]=P(N=0)+W *[W(S(t) ; N-r+1, r] V(N \geq r \geq 1)
$$


If $\lim _{\mathrm{t} \rightarrow \infty} \mathrm{S}(\mathrm{t})=0$ then $\mathrm{S}^{*}(\mathrm{t})$ becomes improper when $\lim _{\mathrm{t} \rightarrow \infty} \mathrm{S}^{*}(\mathrm{t})=\mathrm{P}(\mathrm{N}=0)>0$

The function $\mathrm{S}^{*}(\mathrm{t})$ is $0<\mathrm{S}^{*}(\mathrm{t})<1$ and it is restricted with $\mathrm{N} \geq \mathrm{r} \geq 1$.

The probability function $\mathrm{P}(\mathrm{N}=0)$ is the cure fraction, which is depends only on the distribution of $\mathrm{N}$ irrespective of the value of $\mathrm{r}$. The function $\mathrm{f}^{*}(\mathrm{t})$ is

$$
f^{*}(t)=f(t) W^{*}\left[N\left(\begin{array}{c}
N-1 \\
r-1
\end{array}\right) \times\left[S(t)^{N-r}\right][F(t)]^{r-1} V(N \geq r \geq 1]\right.
$$

Where $f(t)$ is proper density of $F(t)$. It is not possible to measure the value of $N$ and can be modeled through probabilistic terms. However, the value of $\mathrm{N}$ can be specified for $\mathrm{r}$. Now, let $\mathrm{N}$ follows $\operatorname{Poisson}(\theta)$ with a mean $\theta$. So, the cure fraction is defined with

$$
P(N=0)=\exp (-\theta)
$$

The parameter $\theta$ is observed through non-informative prior $\mathrm{g}(\theta)$. It generates the flat prior on $\log (\theta)$.

The value $r=1$, involves that $T=\operatorname{Min}_{1 \leq k \leq N} Y_{k}$ and similarly $r=N$ gives $T=\operatorname{Max}_{1 \leq k \leq N} Y_{k}$

The problem is still exists to specify $\mathrm{r} \mid \mathrm{N}$. If $\mathrm{r} \mid \mathrm{N}$ assumed to follow discrete uniform distribution. Then the equation (5) is

$$
S^{*}(t)=P(N=0)+S(t)(1-P(N=0)
$$

The cure fraction depends on $\mathrm{P}(\mathrm{N}=0)$ with $\mathrm{N} \sim \operatorname{Poisson}(\theta)$ the non-informative prior is set as $\mathrm{g}(\theta)=1 / \theta$.

\section{DIFFERENT MODELS}

\section{1. $\quad$ Model1}

Let $\mathrm{r}=1$, then $\mathrm{T}=\operatorname{Min}_{1 \leq \mathrm{k} \leq \mathrm{N}} \mathrm{Y}_{\mathrm{k}}$ and

$$
P(T \geq t \mid N)=1 .(N=0)+[S(t)]^{N} V(N \geq 1)
$$

It is assumed that $\mathrm{N} \sim \operatorname{Poisson}(\theta)$ with moment generating function $\mathrm{m}(\mathrm{t})$. The cure fraction is defined as

$$
P(N=0)=m(-\infty)
$$

The marginal distribution of $\mathrm{T}$ is obtained with

$$
S^{*}(t)=m[\log S(t)]
$$

\section{2. $\quad$ Model 2}

Let $r=N$, then $T=\operatorname{Max}_{1 \leq k \leq N} Y_{k}$ and the conditional distribution of $T$ given $N$ is expressed as $F(t)=1-S(t)$ with

$$
S^{*}(t)=1+m(-\infty)-m[\log F(t)]
$$

For: $N \sim \operatorname{Poisson}(\theta), S^{*}(t)=1+\exp (-\theta)(1-\exp ((t)))$.

For: $N \sim \operatorname{Geo}(\theta), S^{*}(t)=(1-\theta)+\left[\theta^{2} F(t) /(1-\theta F(t))\right]$ with $1-\exp (-\theta)$ is acure fraction for $0<\theta<1$.

Let the hazard function $h^{*}(t)$ and it can be defined with

$$
\begin{aligned}
m^{\prime}(t) & =\frac{\delta}{\delta t} m(t) \\
h_{1}^{*}(t) & =\frac{m^{\prime}[\log \{S(t)\}]}{m[\log \{S(t)\}]} \text { for model } 1
\end{aligned}
$$




$$
h_{2}^{*}(t)=\frac{m^{\prime}(\log F(t)) f(t)}{[1+m(-\infty)-m(\log F(t)] F(t)} \text { for model } 2
$$

Let

$$
J(t)=h_{1}{ }^{*}(t) / h_{2}{ }^{*}(t)
$$

Here, $J(t)$ is dependent on $S(t)$ and $\theta$. If $\lim _{t \rightarrow 0} J(t)=e^{\theta}$ and $\lim _{t \rightarrow \infty} J(t)=e^{-\theta}$.

the link function $J\left(X^{T} \beta\right)$ is defined as $\theta$. Here $X_{i}$ is covariate of interest for the $\mathrm{i}^{\text {th }}$ individual and $\theta_{i}$ is assumed as mean parameter.

The Model 1 (obtained from equation 11)and Model 2 (obtained from equation 12) are subdivided with [Model 1(a), Model 1(b), Model 1(c), Model 1(d)] and [Model 2(a), Model 2(b), Model 2(c), Model 2(d)] respectively. The assumptions are

Model $1(a)$ and Model $2(a): J\left(X^{T} \beta\right)=\theta_{i} ; \theta_{i}=\theta ; N \sim \operatorname{Poisson}(\theta)$

Model $1(b)$ and Model $2(b): J\left(X^{T} \beta\right)=\theta_{i} ; \theta_{i}=\theta ; N \sim \operatorname{Ber}(\theta)$

Model 1 (c) and Model $2(c): J\left(X^{T} \beta\right)=\theta_{i} ; \theta_{i}=\theta ; N \sim \operatorname{Bin}(K, \theta)$

Model $1(d)$ and Model $2(d): J\left(X^{T} \beta\right)=\theta_{i} ; \theta_{i}=\theta ; N \sim \operatorname{Geo}(\theta)$

The response in the above models is distributed with Weibull distribution but free with value of $N$. These are maintained with cure parameter $\theta$. In this study, the performances of model are compared.

\section{ANALYSIS OF DATA}

Let $\mathrm{Y}$ is the survival time of any subject. The indicator variable $\mathrm{K}=0$ when the subject is cured and $\mathrm{K} \neq 0$ when he/she is non-cured. Let $\mathrm{P}(\mathrm{K}=0)=\mathrm{p}$, and

$\mathrm{P}(\mathrm{K} \neq 0)=1-\mathrm{p}$. Suppose, $\mathrm{F}$ is the cumulative distribution function of the whole population and $\mathrm{F}_{0}$ is the cdf for the non-cured. For $\mathrm{y} \geq 0$.

$$
P(Y \leq y \mid K \neq 0)=F_{0}(y)
$$

Then the $F(y)$ can be defined as

$$
P(Y \leq y / N=1)=0
$$

so,

$$
F_{0}(y)=\frac{F(y)}{1-p}
$$

It is to be noted that $F$ is an improper $\operatorname{cdf}$ with $F(\alpha)<1$.

Suppose, $S(y)=1-F(y)$ and $S_{0}(y)=1-F_{0}(y)$ are the survival function for the corresponding cdfs. Then

$$
S(y)=1-(1-p) F_{0}(y)=p+(1-p) S_{0}(y)
$$

The term $\mathrm{p}$ is substituted with $\exp (-\theta)$. The count variable with observation $\mathrm{N}$ in the population can be assumed with Poission distribution with mean $\theta$.

Let $\mathrm{M}_{\mathrm{i}}$ is the random variable of time for the $\mathrm{i}^{\text {th }}$ individual MELD score.

$$
F(y)=1-S(y)
$$

The time of decline of liver functions is defined with random variable 
426 The Cure Rate Modeling: An Application with Bayesian approach in Liver Cirrhosis Patients

$$
Y=\operatorname{Min}\left\{M_{i}, 0 \leq i \leq N\right\}, \text { whereP }\left(M_{0}=\infty\right)=1
$$

The survival function of $Y$ is defined as

$$
S(y)=P(M E L D \text { score in time } y)
$$

The equation (27) is extended into two parts by

$$
S(y)=\exp (-\theta)+\sum_{k=1}^{\infty} S(y)^{k} \frac{\theta^{k}}{k !} \exp (-\theta)=\exp (-\theta+\theta S(y))=\operatorname{Exp}(-\theta F(y))
$$

as $\theta \rightarrow \infty$, the cure rate function $S(y)$ tends to zero and for $\theta \rightarrow 0$ it tends to 1 . The density function is used for

$$
f(y)=\frac{d}{d y} F(y)
$$

where,

$$
f(y)=\theta f(y) \exp (-\theta F(y))
$$

However $f(y)$ not the proper probability density function and $S(y)$ is not also proper survival function. The hazard function is

$$
h(y)=\theta f(y)
$$

where, $h(y) \rightarrow 0$ at a fast rate as $y \rightarrow \infty$ and $\int_{0}^{\infty} \mathrm{h}(\mathrm{y}) \mathrm{dy}<\infty$. Here, the covariates are modelled through $\theta$ cure rate modelling. This is attractive characteristics of cure rate model. The part with non-cured rate model is designed with

$$
S^{*}(y)=\frac{\exp (-\theta F(y))-\exp (-\theta)}{1-\exp (-\theta)}
$$

$S^{*}(0)=1, S^{*}(\infty)=0$ So that $S^{*}(t)$ is a proper survival function. The survival density for the non-cured population is given

$$
f^{*}(y)=-\frac{d}{d y} S^{*}(y)=\frac{\exp (-(y))}{1-\exp (-\theta)}(y)
$$

and the hazard function for the non-cured population is denoted with

$$
h^{*}(y)=\frac{f^{*}(y)}{S^{*}(y)}=\frac{\exp (-\theta F(y))}{\exp (-(y))-\exp (-\theta)} h(y)=\left(\frac{1}{P(Y<\infty \mid Y>y)}\right) h(y)
$$

The above mentioned equation is extended with $h^{*}(y) \rightarrow \frac{f(y)}{S(y)}$ as $y \rightarrow \infty$, and thus $h^{*}(y)$ converges to the hazard function to the promotion time variable M as $y \rightarrow \infty$. The cure rate model is defined with mixture of cured and non-cured rate model by,

$$
S(y)=\exp (-\theta)+\left(1-\exp (-\theta) S^{*}(y)\right)
$$

The term $\exp (-\theta)$ is used as cured function and $S^{*}(y)$ as non-cured function. In the above mentioned equation $\exp (-\theta)$ is defined with $-x \beta$. The relationship exists with

$\theta=\exp (x \beta)$ for $p \times 1$ vector of covariate of $x$ and regression co-efficient $\beta$ only.

\section{LIKELIHOOD AND POSTERIOR DISTRIBUTION}


In case of $\mathrm{i}^{\text {th }}$ individual, the data is presented as $\mathrm{D}_{\mathrm{i}}=\left(\mathrm{x}_{\mathrm{i}}, \mathrm{t}_{\mathrm{i}}, \mathrm{v}_{\mathrm{i}}\right)$. Here, $\mathrm{x}_{\mathrm{i}}$ is the covariate of interest, $t_{i}$ is the failure time and $v_{i}$ the indicator of failure.

The likelihood function for the $\mathrm{i}^{\text {th }}$ individual of data is defined with

$$
\left.\left.L(\theta, \varphi \mid D)=\left(\prod_{i=1}^{n} S\left(\frac{y_{i}}{\varphi}\right)^{N_{i}-v_{i}}\right)\left(N_{i} f\left(y_{i} \mid \varphi\right)\right) v_{i}\right) * \exp \left\{\sum_{i=1}^{n} N_{i} \log (\theta)-\log \left(N_{i} !\right)\right)-n \theta\right\}
$$

The density function of weibull is

$$
f\left(y_{i} \mid \varphi\right)=\alpha y^{\alpha-1} \exp \left\{\lambda-y^{\alpha} \exp (\lambda)\right\}
$$

The joint non-informative prior $\pi(\beta, \varphi) \propto \pi(\varphi)$ is applied for the function $f\left(y_{i} \mid \varphi\right)$ of Weibull distribution. The parameter $\varphi$ is derived from the parameters of the equation (15) i.e. $(\alpha, \lambda)^{\prime}$. The parameters in the function $\pi(\beta, \varphi)$ are independent and $\pi(\beta)$ is assumed with uniform prior. Further the function $\pi(\varphi)$ is generated with

$$
\pi(\varphi)=\pi\left(\varphi \mid \delta_{0}, \tau_{0}\right) * \pi(\lambda)
$$

and

$$
\pi\left(\alpha, \delta_{0}, \tau_{0}\right) \propto \alpha^{\delta_{0}-1} \exp \left(-\tau_{0} \alpha\right)
$$

Here, $\delta_{0}$ and $\tau_{0}$ are the hyper-parameters and the posterior distribution of $(\beta, \varphi)$ based on the observed data is

$$
D_{o b s}=(n, y, X, v)
$$

with

$$
\pi\left(\beta, \varphi \mid D_{\text {obs }}\right) \propto\left(\sum L(\beta, \varphi \mid D)\right) * \pi\left(\alpha \mid \delta_{0}, \tau_{0}\right) * \pi(\lambda)
$$

The posterior mean and Highest Posterior Estimates has been conducted through MCMC simulation. The likelihood used to compute the posterior is

$$
\pi\left(\beta, \varphi, a_{0} \mid D_{o b s}\right) \propto\left[\sum L\left(\beta, \varphi \mid D_{0}\right)\right]^{a_{0}} * \pi_{0}(\beta, \varphi) a_{0} \gamma_{0}-1\left(1-a_{0}\right)^{\lambda_{0}-1}
$$

The beta prior is assumed for $a_{0}$. The normal prior with mean 0 and variance 0.001 is applied for $\lambda$ and $\gamma$ parameter. The function $L(\beta, \phi \mid D)$ is observed with data likelihood of $D$. The results are given in next section.

\section{SIMULATION STUDY}

In view of the objective stated in Introduction section, the models are applied to estimate the parameter of interest. The developed models are used to compare the estimated parameters of interest. The iteration number was fixed with 5000 samples. The results are given in the Table 4 . Based on the simulation, it is concurred that the relative bias is not enormous. The maximum relative bias is observed by $30 \%$. It shows that the proposed approach turn out estimates near to the true parameters values. It can be concluded that the proposed approach is consistent and provides logical output.

\section{MODEL COMPARISON}


The selection of best model is important in any statistical inference. The model selection must be based on scientific impact and repetitive in ideal condition in other study. The likelihood given above can be different for the models to serve the real assumption and requirement of hazard and survival function. There are several types of model selection procedures with selection tools like Bayes factor (Robert et al., 1995), BIC, AIC and DIC. The DIC is selected for the best fitted model mentioned in equation (17) to (20).

The DIC is based on the posteriors by,

$$
D I C=D(\bar{y})+P D
$$

The term PD is applied to measures the complexity of the model. It is defined as

$$
P D=E_{\gamma \mid \bar{x}}\left[-2 \log L\left(\frac{\bar{x}}{\gamma}\right)\right]+2 \log L\left(\frac{\bar{x}}{\gamma}\right)
$$

and

$$
D(\gamma)=E_{\gamma / \bar{x}}\left[-2 \log L\left(\frac{\bar{x}}{\gamma}\right)\right]
$$

$\bar{\gamma}$ is the estimated value of the $\gamma$. It is the posterior mean of the posterior median [28]. The model has the result with smallest DIC is considered as best fitted. The software WINBUGS version 2.14 is explored to compute the DIC value.

\section{ANALYSES OF LIVER CIRRHOSIS DATA}

Table 1 contains the baseline and demography characteristics of liver cirrhosis patient in treated and control group. The mean age of liver cirrhosis patient in treatment group is 48.6 with standard deviation (SD) 9.38 whereas in control group, the mean age is 49.85 with SD 11.06. The distribution of male in treated and control group is $10(12.05 \%)$ and $70(76.09 \%)$ respectively. Similarly the distribution of female in treated and control is $73(87.95 \%)$ and $22(23.91 \%)$ respectively. The mean height of liver cirrhosis patient in treatment group is 164.89 with SD 4.46 whereas in control group, the mean height is 165.38 with SD 5.84. The mean weight of liver cirrhosis patient in treatment group is 65.71 with SD 5.24 whereas in control group, the mean weight is 69.51 with SD 8.87. The mean Respiratory Rate (RR) of liver cirrhosis patient in treatment group is 25.99 with SD 16.59 whereas in control group, the mean RR is 21.43 with SD 1.62. The mean Hear Rate (HR) of liver cirrhosis patient in treatment group is 72.89 with SD 15.61 whereas in control group, the mean HR is 77.22 with SD 2.08. Figure 1-3: Demonstrates the distribution of liver cirrhosis patients surviving over the study duration using Kaplan Meier plot. Figure 4: Demonstrates the distribution of transformed MELD scores at different arbitrary values of lambda. To illustrate the methodology, the data from Liver cirrhosis clinical trial considered. The covariates are age(x1), sex(x2) (male, female), and MELD Score(x3). Table 2 gives statistical summaries for the covariates for this subset of 94 subjects are used in the analysis. In all of the computations, all the covariates are standardized to have mean 0 and standard deviation 1 in order to improve the convergence of the MCMC algorithm. Specially, standardizing the covariates greatly reduces the correlation between the intercept term and the other regression coefficients. The regression co-efficient of covariate age refers that there is positive impact of age on cure rate for patients in treated group as compare to control group i.e. if there is an increase of one year then it 
is expected that there may be a chance of increase in cure rate by 0.21 in treated group as compare to control group with $95 \%$ credible interval $(0.15,0.24)$. The interpretation of age can also be given in odds ratio $(\exp (\beta A g e=1.23))$. The subject becomes older by one year the odds of getting cure in treated group is more as compare to control group. The similar interpretation can be given for $\operatorname{sex}(\exp (\beta$ sex $=1.59))$. The male patients have 1.59 times more odds of get- ting cured as compare to female patient when treated with study therapy. The kappa value of 5.78 indicates that there is a positive agreement of covariates (age, gender and MELD score) on liver cirrhosis patients for better cure for treated group as compare to control group with 95\% credible interval $(2.58,10.34)$.The estimated Posterior Medians of different Models is provided in Table 3. The Goodness-of-Fit for model on Liver Cirrhosis Dataset is provided in Table 4.

\section{MODEL PERFORMANCE}

A total of 10,000 samples with 1000 refreshment are generated. The effect of MELD score are observed for each models to fit the models with 1(a), 1(c), 1(d), and 1(e) and consequently 2(a), 2(c), 2(d), and 2(e). The continuous variables are assumed to be generated from $N(0,1)$ In model

$1(\mathrm{~d})$ and $2(\mathrm{~d})$ it is assumed that $J\left(X^{T} \beta\right)=2$ and $\beta_{1}=1$. In case of model 1(a), 1(c) and 2(a) to 2 (c) the $\beta=(3,1)$ and $\rho=1$ is considered for model iterations. The initial value of parameters is based on optimum value observation through simulation study. The histogram of MELD scores from the simulation studies are given in figure4.The MCMC iteration provides advantage over uncertainty of the models. This parameter appeared to be well identified in our MCMC computations. The model 1(a) acquired 95\% posterior credible interval for $\mathrm{P}(\mathrm{r}=1)$ about $-1.21(-$ $1.63,-1.02)$ with assumption of having $\mathrm{P}(\mathrm{r}=1)=\mathrm{P}(\mathrm{r}=\mathrm{N})=1 / 2$. Sex, the interval was $-0.11(-0.19$,0.03 ) in Model 2(a). Model 1(a), 1(b), 1(d) are found perform inferior than model 1(c). Model 2(c) seems to be performed better than model 2(a), 2(b) and 2(d). The analysis of MELD score is illustrated methods on discussed model over all observed patients in the clinical trials. It is performed to compare between two treatment groups. The comparison between cases and control are performed with Kaplan Meier estimators. The product limit comparisons are given in figure 1 to 3 . The cure fractions observed through models are given in tables 4. The Kaplan-Meier estimates between treatment and control is also produced after adjustment with sex. Among male patients the control groups worked better than cases (figure2). The overall survival among control group found better than cases (figure 3). The distributions of transformed MELD score at different arbitrary values of lambda are given in figure 4 . The relative biases are given in table 4 for different parameters. It shows that the maximum bias observed for $\beta_{2}$ in model 2 (b) and minimum $\beta_{3}$ in model 1(a). The best fitted model is 1(c) with DIC value 230.14. The DIC's for model 1(a), 1(b) and 1(d) are 243.52, 237.65 and 250.61. Whereas, the DIC observed for model 2(a), 2(b) and 2(c) are 273.12, 247.91, 242.35 and 265.79. It shows that the model 2(c) is best among models 2(a-d) (Table 5). The level of harm due to alcohol in the population can be detected through liver cirrhosis prevalence (Leon et al., 2006). The data are considered generated from Phase three clinical trial in which liver cirrhosis subjects were undergone with HFLPC for 36 months. The dataset includes 104 subjects, of whom 62 are male. In count, the covariate of interest MELD score is used as indicator ( up to normal $=0$ other wise 1 ) for each individual The descriptive statistics with KaplanMeier curve are given in the Figure 1 to 3 . Table 3 gives the posterior median estimates of MELD scores for the different models of the liver cirrhosis data set. The covariates included are a geat baseline observation, sex (male or female), and MELD score (normal or not). As per model 2 from section 3, the parameters affect the cure probability, with positive estimates. 


\section{DISCUSSION}

This paper has explored the risk of death due to liver transplantation in Cirrhosis patients including time-dependent effects terms. The Bayesian prior assumption is applied to formulate the posterior mean from the cure rate modelling. This work is contributed with exploration of cure rate modelling in liver cirrhosis patients. The MELD score is considered as response in the cure rate modelling. The illustrated method can also be suitable in other experimental study. The cure rate model given in this study is to explore the data on liver cirrhosis where significant proportions of patients are cured. The cure rate model is an alternative choice of Cox proportional regression model. The Cox proportional model is simple because it is free with parametric assumption. However, the cure rate model is useful to stratify the patients into two groups and to compare the performance between cure and non-cured groups. An application of the model to the data from the liver cirrhosis patients under gone to the treatment with HPCLC for one year gives the important information about drug effect. The study could be extended with inclusion of other related covariates. The model proposed in this paper assumed that the covariates affect only the probability of being cured. A more general model may be proposed that also includes the effects of covariates on the failure time distribution. An $\mathrm{R}$ and Winbugs package are available for download at the web site

http://cran.r-pro ject.org/

and

http://www.mrc-bsu.cam.ac.uk/bugs/winbugs/contents.shtml.

Using these programs, cure rate modelling can be applied in situation similar to those presented in this paper. The applied method may also be suitable in other experimental study. The model can be valid in the small size data set. The regression co-efficient of covariate (age) refers that there is positive impact of age on cure rate for patients in treated group as compared to control group The male patients have higher odds of getting cured as compared to female patient when treated with study therapy. The kappa estimates indicates that there is a positive agreement of covariates (age, gender and MELD score) on liver cirrhosis patients for better cure for treated group as compare to control group. In this data we found that Model 1 (c) and Model 2(c) give precise estimates of posterior median in comparison with all other models i.e. these two models have very small DIC values among all other models (Table 5).

\section{ACKNOWLEDGEMENTS}

The authors would like to express sincere gratitude to two anonymous referees for their cautious reading and constructive suggestions which led to improvement over the earlier versions of the manuscript.

The authors also gratefully acknowledge the time and expertise devoted in reviewing paper by the advisory reviewer and their advice for promptness in dealing with manuscript. 


\section{REFERENCE}

[1] Malinchoc, M., Kamath, P.S., Gordon F.D., Peine C.J., Rank J, Borg P.C (2000). A model to predict poor survival in patients undergoing transjugular intrahepatic portosystemic shunts. Hepatology 31, 864-71.

[2] Farewell, V. T. (1982), The use of mixture models for the analysis of survival data with longterm survivors.Biometrics38, 1041-1046.

[3] Gamel, J. W., McLean, I. W. and Rosenberg, S. H. O. (1990). Proportion cured andmean log survival time as functions of tumor size O.Statistics in Medicine 9, 999-1006.

[4] Yamaguchi, K (1992).Accelerated failure-time regression model with a regression model for the surviving fraction: an application to the analysis of 'permanent employment' in Japan. Journal of the American Statistical Association87, 284-292.

[5] Cancho, V.G. and Bolfarine, H (2001). Modeling the presence of immunes by using the exponentiated -Weibull model.Journal of Applied Statistics 28, 659 - 671.

[6] Taylor, J. M. G (1995). Semiparametric estimation in failure time mixture models, Biometrics51, 899-907.

[7] Nelson, W (1982). Applied life data analysis, John Wiley \& Sons, New York

[8] Boag, J. W. O. (1949). Maximum likelihood estimates of the proportion of patients cured by cancer therapy O. Journal of the Royal Statistical Society 11, 53.

[9] Struthers, C. A. and Farewell, V. T(1989). A mixture model for time to AIDS data with left truncation and an uncertain origin.Biometrika76, 814-817.

[10] Maller, R, and Zhou X (1996). Survival analysis with long-term survivors, John Wiley \& Sons, Inc., New York.

[11]Jones, D. R., Powles, R. L., Machin, D. and Sylvester, R. J (1981). On estimating the proportion of cured patients in clinical studies O. Biometrie-Praximetrie21,1-11 .

[12] Yu, B., Tiwari R.C., Cronin K.A., Feuer E.J. (2004): Cure fraction estimation from the mixture cure models for grouped survival data. Statistics in Medicine 23(11), 1733-1747.

[13] Kamath, P.S., Wiesner, R.H., Malinchoc, M., Kremers, W., Therneau, T.M., Kos-berg, C.L., D'Amico, G., Dickson, E.R., Kim, W.R. (2001). A model to predict survival in patients with end-stage liver disease.Hepatology 33, 464-70.

[14]Wiesner, R.H., McDiarmid, S.V., Kamath, P.S., et al (2001). MELD and PELD: application on survival models to liver allocation. Liver Transplantation7, 567-80.

[15]Freeman, R.B., Jr, Wiesner, R.H., Harper, A. et al (2002). UNOS/OPTN Liver Disease Severity Score, UNOS/OPTN Liver and Intestine, and UNOS/OPTN Pediatric Transplantation Committees. The new liver allocation system: moving toward evidence-based transplantation policy, Liver Transplantation 8, 851-8.

[16] Everson, G.T. (2003). MELD: The answer or just more questions? Gastroenterology 124, 2514.

[17]Freeman, R.B.(2005). MELD: the holy grail of organ allocation ? J Hepatol42, 16-20.

[18]Cholongitas, E., Senzolo, M., Triantos, C., et al. MELD is not enough-enough of MELD? J Hepatol 42, 475-7.

[19]Durand, F., Valla, D. (2005). Assessment of the prognosis of cirrhosis: Child-Pugh versus MELD. J Hepatol 42, S100-7.

[20] Yakovlev, A. Y. (1996). Threshold Models of Tumor Recurrence,Mathematical and Computer Modelling 23, 153-164.

[21] Yakovlev, A. Y., Asselain, B., Bardou, V. J., Fourquet, A., Hoang, T., Rochefordiere, A., and Tsodikov, A. D. (1993), "A Simple Stochastic Model of Tumor Recurrence and Its Application to Data on Premenopausal Breast Cancer," in Biometrie et Analyse de DonneesSpatio-Temporelles 12, eds. B. Asselain, M. Boniface, C. Duby, C. Lopez, J. P. Masson, and J. Tranchefort, Rennes, France: SocitFranaise de Biomtrie, 66-82.

[22] Yakovlev, A. Y. and Tsodikov, A. D. (1996) Stochastic Models of Tumor Latency and Their Biostatistical Applications, Singapore, World Scientific. 
[23]Chen, M. H., Ibrahim, J. G. and Sinha, D (1999). A New Bayesian Model forSurvival Data With a Surviving Fraction.Journal of the American Statistical Association 94, 909-919.

[24]Lambert, P.C., Thompson, J.R., Weston, C.L., Dickman, P.W. (2007). Estimating and modeling the cure fraction in population-based cancer survival analysis. Biostatistics 8(3), 576-594.

[25] Royston, P. (2001). Flexible parametric alternatives to the Cox model, and more. The Stata Journal 1, 1-28.

[26] Nelson, C.P., Lambert, P.C., Squire, I.B., Jones, D.R (2007). Flexible parametric models for relative survival, with application in coronary heart disease. Statistics in Medicine 26(30), 5486-5498.

[27]Robert, E. Kass, Adrian, E., Raftery (1995). Bayes Factors.Journal of the American Statistical Association 430, 773-795.

[28] Spiegelhalter, D. J., Nicola, G. B., Bradley, P. C. and Angelika, V. D. L. (2002).Bayesian measures of model complexity and fit. Journal of Royal statistical Society B 64(4),583-914 
Table 1: Descriptive statistics about baseline observations of the patients

\begin{tabular}{|c|c|c|c|}
\hline \multicolumn{2}{|c|}{ Parameters } & $\begin{array}{l}\text { Treatment group } \\
\text { Mean (SD) }\end{array}$ & $\begin{array}{l}\text { Control Group } \\
\text { Mean (SD) }\end{array}$ \\
\hline Age & & $48.60(9.38)$ & $49.85(11.06)$ \\
\hline \multirow[t]{2}{*}{ Gender } & Male & 10 (12.05\%) & 70 (76.09\%) \\
\hline & Female & 73 (87.95\%) & 22 (23.91\%) \\
\hline Height & & $164.89(4.46)$ & $165.38(5.84)$ \\
\hline Weight & & $65.71(5.24)$ & $69.51(8.78)$ \\
\hline Respiratc & ry rate (RR) & $25.99(16.59)$ & $21.43(1.62)$ \\
\hline Heart Ra & & $72.89(15.61)$ & $77.22(2.08)$ \\
\hline
\end{tabular}

Table 2: The posterior estimates of covariates in cured rate modelling

\begin{tabular}{lllll}
\hline Parameter & Mean & SD & $2.5 \%$ & $97.5 \%$ \\
\hline BAge & 0.21 & 0.03 & 0.15 & 0.24 \\
$\mu$ & 4.10 & 0.30 & 3.87 & 4.25 \\
$\beta$ Sex & 0.47 & 0.13 & 0.32 & 0.63 \\
\hline
\end{tabular}


434 The Cure Rate Modeling: An Application with Bayesian approach in Liver Cirrhosis Patients

Table 3: Posterior Median of Different Models

$\begin{array}{ll}\text { Model } 1 & \text { Model 2 }\end{array}$

Parameter

(a)

(b)

(c)

(d)

(a)

(b)

(c)

(d)

\begin{tabular}{|c|c|c|c|c|c|c|c|c|}
\hline Intercept & $-1.21(-1.63,-1.02)$ & $-0.92(-1.10,-0.76)$ & $-0.05(-0.19,0.11)$ & $-1.03(-1.24,-0.89)$ & $-2.08(-2.21,-1.93)$ & $-0.92(-1.05,-0.79)$ & $-.98(-1.13,-.78)$ & $-1.23(-1.32,-1.14)$ \\
\hline Age & $0.13(-0.63,0.15)$ & $-0.17(-0.29,-0.09)$ & $-0.23(-0.32,-0.12)$ & $-0.27(-0.43,-0.12)$ & $-0.10(-0.25,0.07)$ & $-0.19(-0.49,0.02)$ & $-0.20(-0.39,0.02)$ & $-0.17(-0.34,-0.01)$ \\
\hline Sex & $-0.22(-0.41,-0.02)$ & $-0.16(-0.23,-0.11)$ & $-0.06(-.11,-0.03)$ & $-0.08(-.12,-0.02)$ & $-0.11(-.19,-0.03)$ & $-0.18(-0.29,-0.03)$ & $-0.12(-0.22,-0.01)$ & $-0.03(-0.2,0.11)$ \\
\hline $\begin{array}{l}\text { MELD } \\
\text { score }\end{array}$ & $-0.19(-0.39,0.03)$ & $-0.21(-0.41,-0.09)$ & $-0.07(-.21,0.11)$ & $-0.17(-0.31,0.02)$ & $-0.16(-0.29,0.01)$ & $-0.8(-0.32,0.11)$ & $-0.09(-0.23,0.02)$ & $-0.14(-0.40,0.03)$ \\
\hline$\eta$ & & & & & $-0.12(-0.43,0.03)$ & & & \\
\hline $\exp (-\theta)$ & $0.42(0.75,0.21)$ & $0.39(0.69,0.06)$ & $0.41(0.77,0.01)$ & $0.45(0.71,0.02)$ & $0.31(0.69,0.01)$ & $0.44(0.83,0.02)$ & $0.37(0.79,0.07)$ & $0.36(0.69,0.08)$ \\
\hline$\theta$ & $1.43(1.59,1.34)$ & $1.21(1.33,1.14)$ & $1.39(1.51,1.29)$ & $1.15(1.32,1.14)$ & $1.53(1.69,1.41)$ & $1.27(1.35,1.14)$ & $1.41(1.57,1.30)$ & $1.23(1.39,1.21$ \\
\hline
\end{tabular}


Table 4: The Goodness-of-Fit for the Liver Cirrhosis Dataset

\begin{tabular}{|c|c|c|c|}
\hline & Parameters & Actual Estimates & Relative Bias \\
\hline \multirow[t]{4}{*}{ Model1(a) } & $\beta 0$ & 1.12 & 1.10 \\
\hline & $\beta 1$ & 1.92 & 1.83 \\
\hline & $\beta 2$ & 1.20 & 1.04 \\
\hline & $\beta 3$ & 2.79 & 2.81 \\
\hline \multirow[t]{4}{*}{ Model1(b) } & $\beta 0$ & 1.13 & 1.17 \\
\hline & $\beta 1$ & 1.89 & 1.93 \\
\hline & $\beta 2$ & 1.23 & 1.08 \\
\hline & $\beta 3$ & 2.72 & 2.77 \\
\hline \multirow[t]{4}{*}{ Model1(c) } & $\beta 0$ & 1.08 & 1.11 \\
\hline & $\beta 1$ & 1.86 & 1.91 \\
\hline & $\beta 2$ & 1.32 & 1.54 \\
\hline & $\beta 3$ & 2.68 & 2.61 \\
\hline \multirow[t]{4}{*}{ Model1(d) } & $\beta 0$ & 1.12 & 1.15 \\
\hline & $\beta 1$ & 1.69 & 1.87 \\
\hline & $\beta 2$ & 1.27 & 1.13 \\
\hline & $\beta 3$ & 2.70 & 2.72 \\
\hline \multirow[t]{4}{*}{ Model2(a) } & $\beta 0$ & 1.11 & 1.10 \\
\hline & $\beta 1$ & 1.85 & 1.81 \\
\hline & $\beta 2$ & 1.29 & 1.11 \\
\hline & $\beta 3$ & 2.58 & 2.67 \\
\hline \multirow[t]{4}{*}{ Model2(b) } & $\beta 0$ & 1.15 & 1.12 \\
\hline & $\beta 1$ & 1.75 & 1.81 \\
\hline & $\beta 2$ & 1.18 & 1.49 \\
\hline & $\beta 3$ & 2.85 & 2.81 \\
\hline \multirow[t]{3}{*}{ Model2(c) } & $\beta 0$ & 1.10 & 1.11 \\
\hline & $\beta 1$ & 1.89 & 1.95 \\
\hline & $\beta 2$ & 1.31 & 1.43 \\
\hline
\end{tabular}


436 The Cure Rate Modeling: An Application with Bayesian approach in Liver Cirrhosis Patients

\begin{tabular}{llll}
\hline & $\beta 3$ & 2.75 & 2.71 \\
Model2(d) & $\beta 0$ & 1.13 & 1.07 \\
& $\beta 1$ & 1.81 & 1.89 \\
& $\beta 2$ & 1.19 & 1.02 \\
& $\beta 3$ & 2.65 & 2.80 \\
\hline
\end{tabular}


Table 5: DIC values for different Models

\begin{tabular}{lc}
\hline Models & DIC values \\
\hline Model 1(a) & 243.52 \\
Model 1(b) & 237.65 \\
Model 1(c) & 230.14 \\
Model 1(d) & 250.61 \\
Model 2(a) & 273.12 \\
Model 2(b) & 247.91 \\
Model 2(c) & 242.35 \\
Model 2(d) & 265.79 \\
\hline
\end{tabular}


438 The Cure Rate Modeling: An Application with Bayesian approach in Liver Cirrhosis Patients

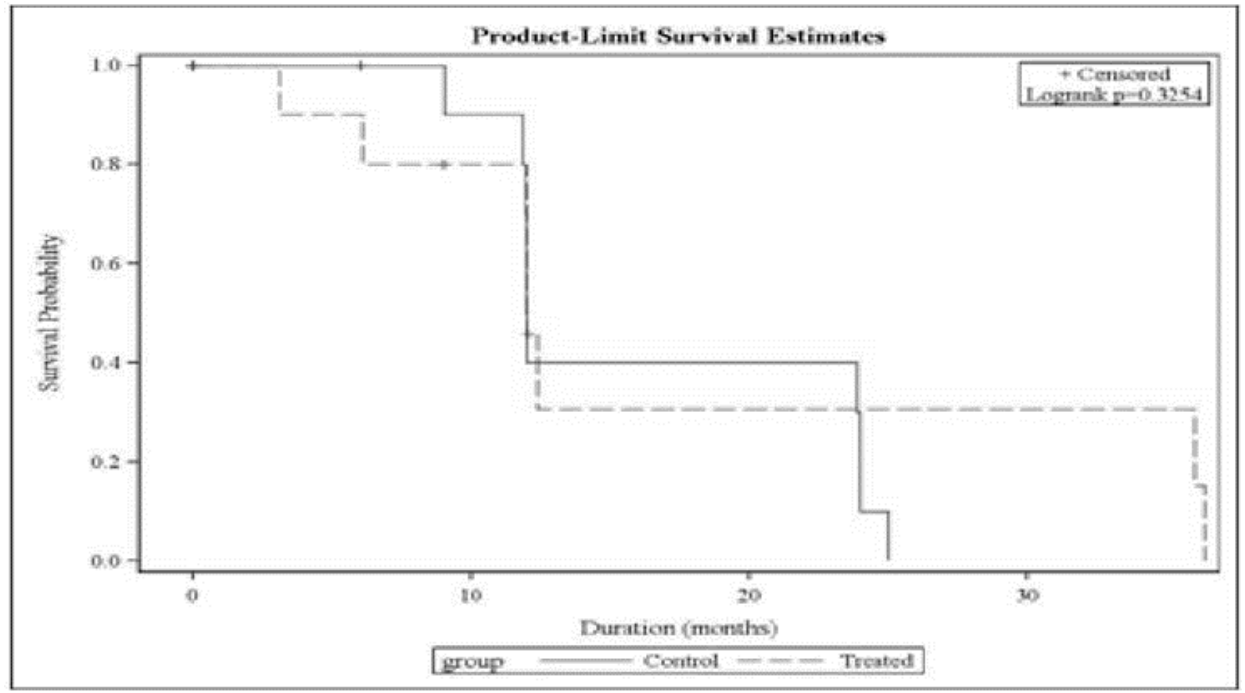

Figure 1: Demonstrate the distribution patients surviving using Kaplan Meier plot in Female group. 


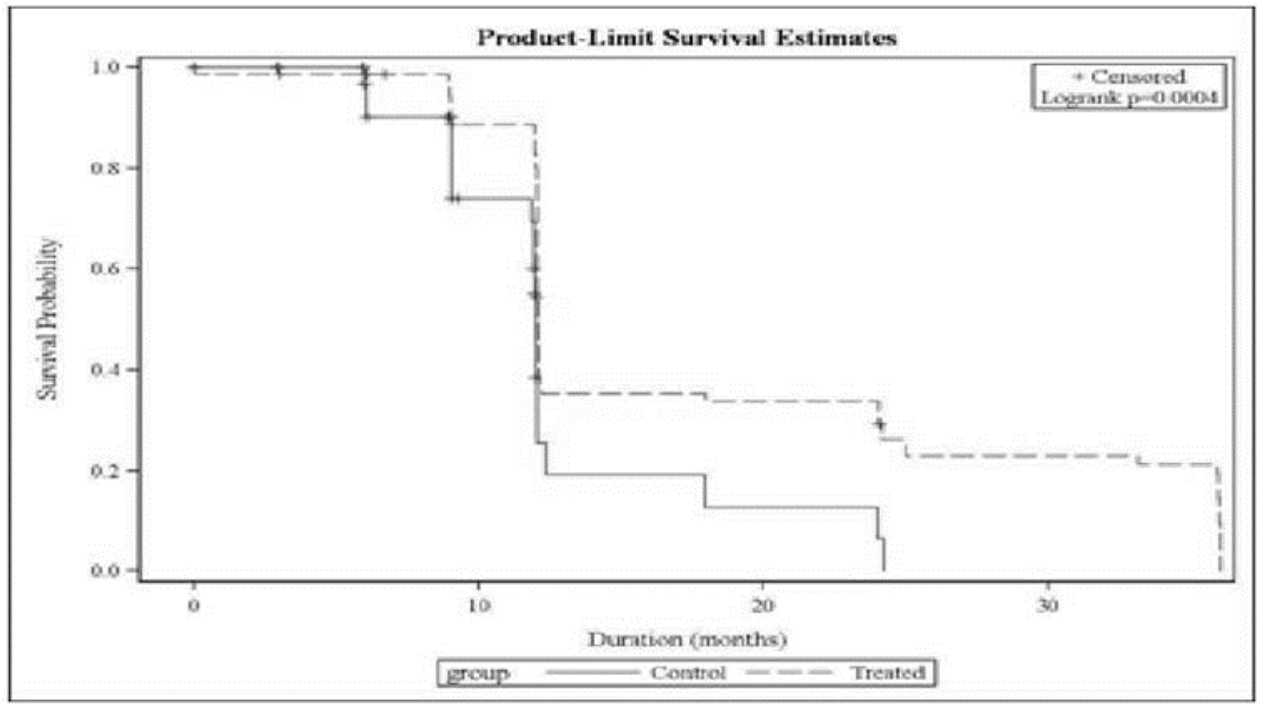

Figure 2: Demonstrate the distribution patients surviving using Kaplan Meier plot in Male group. 
440 The Cure Rate Modeling: An Application with Bayesian approach in Liver Cirrhosis Patients

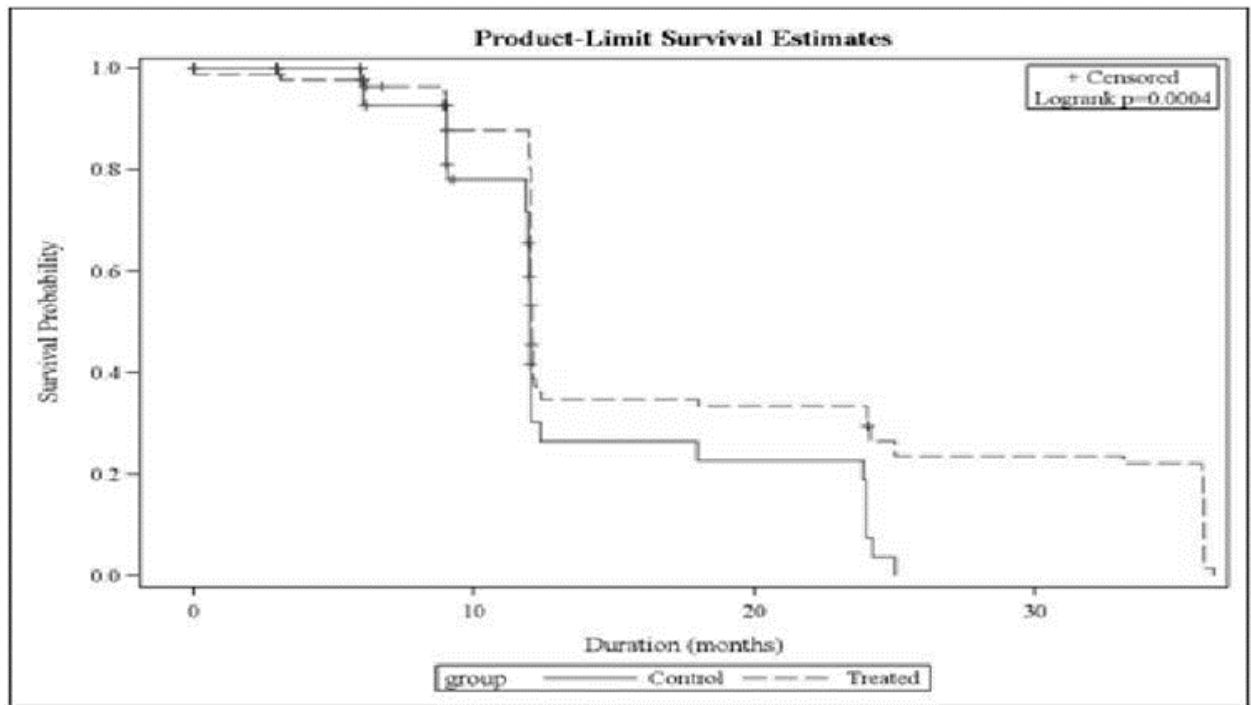

Figure 3: Demonstrate the distribution patients surviving over the study duration using Kaplan Meier plot. 


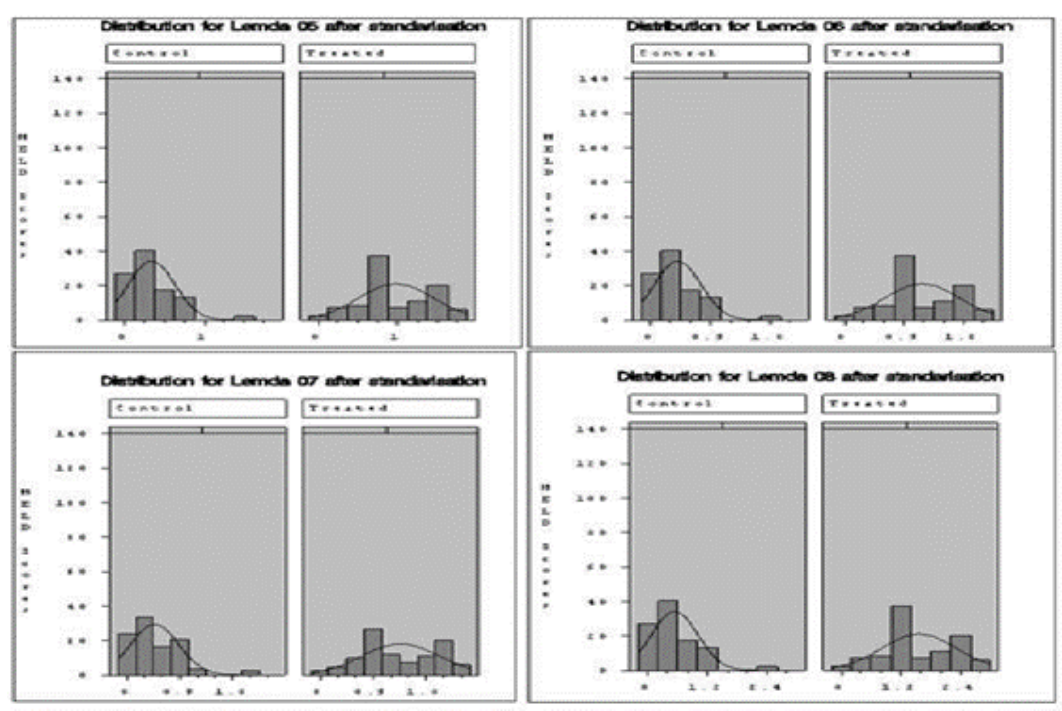

Figure 4: Demonstrate the distribution of transformed MELD scores at different arbitrary values of lambda. 
442 The Cure Rate Modeling: An Application with Bayesian approach in Liver Cirrhosis Patients 